\title{
Original Research \\ A Study of Downwash Effects on Flow and Dispersion Processes around Buildings in Tandem Arrangement
}

\author{
Renata Gnatowska* \\ Institute of Thermal Machinery, Czestochowa University of Technology \\ Armii Krajowej 21, 42-201 Czestochowa, Poland \\ Received: 10 February 2015 \\ Accepted: 22 March 2015
}

\begin{abstract}
Air pollution is one of the world's most important problems. Our paper presents and discusses the relationship between a structure of a flow field among neighboring buildings immersed in a boundary layer, and characteristics of pollutant dispersion in that area. The flow and dispersion of gases emitted by a point source located in front of the buildings also were determined by numerical model with the use of a commercial CFD code (Ansys FLUENT). This paper presents the results of a complex research program aimed at understanding the character of the flow field around groups of buildings in urban areas and the unsteady phenomena resulting from the character of the wind or from the interference of the wake flow connected with the vortexshedding process. The results showed that it is the factor-affecting process of the dispersion of pollutants in the built-up area that is more complex than the propagation of small parts explained on the basis of the processes of advection and turbulent diffusion.
\end{abstract}

Keywords: numerical modeling, buildings arrays, wind tunnel experiment, pollutant dispersion

\section{Introduction}

Modelling of air pollution is currently a leading subject in many research institutions, and it is still new in Eastern European countries. This is mainly forced by current and future EU regulations, which concentrate on protection of the natural environment, and the safety and health of people being exposed to pollutant dispersion processes. Despite the fact that modelling of the turbulence seems to be well covered by the existing turbulence models, a detailed understanding of all interlinked phenomena connected to flow turbulence/dispersion interactions is still far from being complete, though considerable progress has been made in recent years. Literature data as well as my own research show that in strongly unsteady flows the

*e-mail: rgnatowska@gmail.com modelling of turbulence atmospheric flow with pollutants is of primary importance and sometimes is regarded as more important than chemical kinetics of reactions.

The problem of pollution dispersion throughout the atmospheric boundary layer has grown in importance since human activity has become so intense that it has started having considerable impact on the natural environment. The level of concentration of pollutants has escalated - particularly in urban areas - and it impacts their inhabitants [1-5].

Ensuring adequate air quality requires proper aeration of these areas. Its efficiency depends mainly on wind direction, configuration of buildings, and locations of emissions sources. The process of pollution dispersion is mainly influenced by mechanisms of mass diffusion, caused by concentration gradients and advection, which transfers pollutants in flow direction through mean air movement. An important role also is played by turbulent transport 
processes [6]. Improvement in air quality on a local scale and limitation of the effect of pollution on human health requires consideration of all the listed factors. At the present time, CFD is widely employed to study the flow field, pollutant dispersion, and its prediction around buildings [7, 8]. In some earlier published papers [9-11], RANS and LES predictions for plume dispersion have been compared. An important role in increasing knowledge about dispersion processes that occur in the atmosphere is played by the investigations carried out inside wind tunnels [6]. They also deliver data necessary for verification of the results obtained by means of numerical modeling. During model testing of environmental aerodynamics, actual shapes of buildings are typically replaced with their simplified versions (cuboids). This paper is related to the wide and complex theme of environmental protection. The study case refers to the dispersion of pollutants in the vicinity of two buildings in tandem arrangement. A few results that have been obtained on the computer simulation are presented and commented upon, underlining the advantages, restrictions, and limitations of the studied problem.

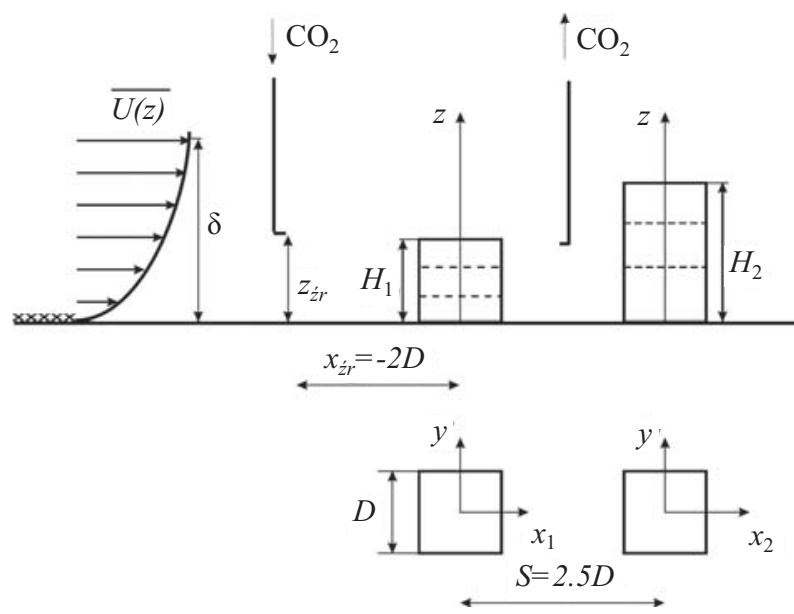

Fig. 1. Schematic presentation of the set-up and nomenclature.

\section{Methods of Analysis}

The program of this study consists of: wind-tunnel measurement of the mean concentration profiles in the interbody gap for different body "immersions" in boundary layer and comparison of concentration field with aerodynamic characteristics (obtained as a result of numerical simulation performed in ITM CzUT). Within the framework of the numerical part of the study the three-dimensional steady and unsteady RANS simulations have been carried out using a commercial CFD code, Ansys FLUENT, with the $\mathrm{RNG}$ version of a $\mathrm{k}-\varepsilon$ turbulence model. According to the literature $[12,13]$, this model is widely used for flows in a build environment. Numerical experiments were comprised of modeling of wind conditions in flow approaching cuboids in tandem arrangements and surface wall shear stress. The experiments were carried out in an open-circuit wind tunnel at the Institute of Thermal Machinery of Czestochowa University of Technology.

All the measurements were carried out for the Reynolds number $\mathrm{Re}_{\mathrm{D}}=3.4 \times 10^{4}$ based on free stream velocity $\mathrm{U}_{\infty}=13$ $\mathrm{m} / \mathrm{s}$ and cube width $\mathrm{D}=0.04 \mathrm{~m}$. The modelled objects have been placed in the boundary layer formed over the terrain of moderate roughness under wind conditions characterized by the mean velocity profile described by the power low $U(z)=U_{0}(z / \delta)^{\alpha}$, where $\delta$ is the depth of boundary layer and $\alpha=0.18$ is the power law exponent, which corresponds to the velocity profile for suburban environments with low vegetation.

Fig. 1 presents the geometries of the analyzed cases of two obstacles, where $\mathrm{H}_{1} / \mathrm{H}_{2}=0.6$ describes their height ratio and $\mathrm{S} / \mathrm{D}=2.5$ the distance between them.

The source of carbon dioxide emissions used as a tracer gas during the investigations was a brass pipe with inner diameter $3 \mathrm{~mm}$, located before the windward object at a distance of $1.5 \mathrm{D} . \mathrm{CO}_{2}$ flow rate was maintained at constant level $Q=51 /$ minute, which produced output speed of

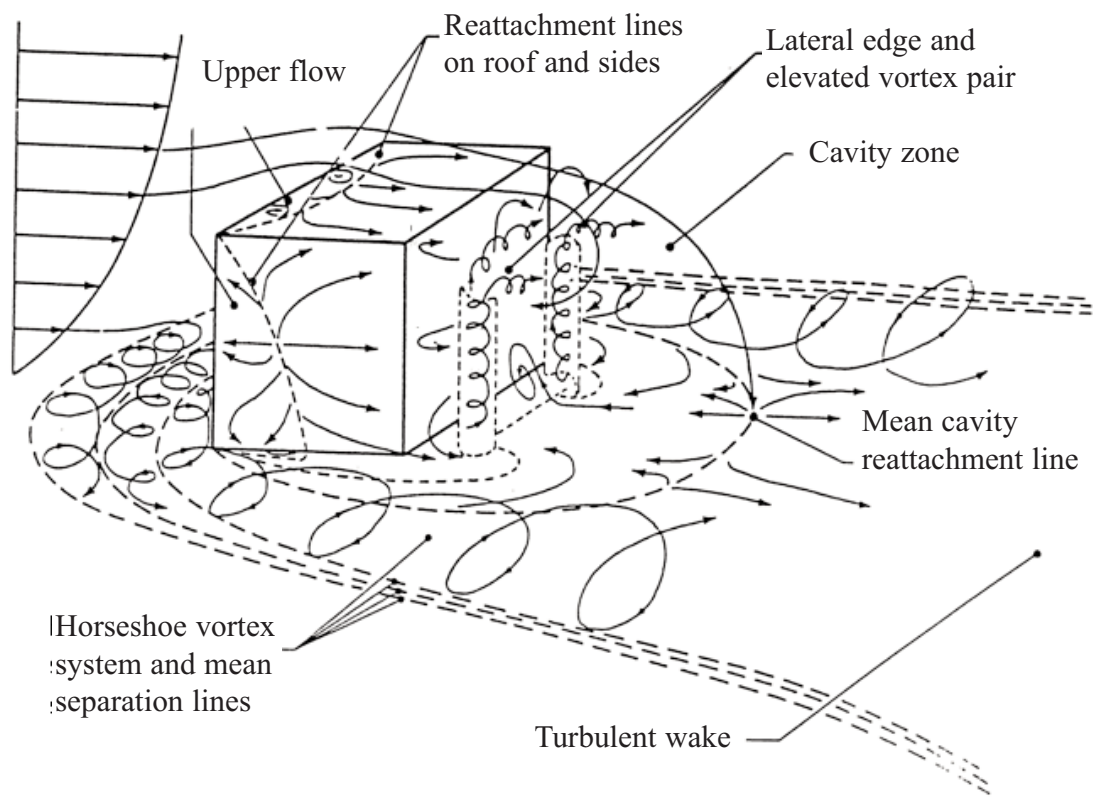

Fig. 2. Model of flow near a sharp-edged building normal to deep boundary layer [9]. 
$U_{\mathrm{CO}_{2}}=11.8 \mathrm{~m} / \mathrm{s}$. In order to measure mean concentration of tracer gas a Guardian plus $\mathrm{CO}_{2}$ analyzer was used. Measurement probe in the form of aluminium pipe with inner diameter of $2.6 \mathrm{~mm}$ was connected with analyzer inlet by means of supple pipe. Location of the source and the measurement probe in relation to the investigated arrangement of cuboids as well as the assumed coordinate system are pre- sented in Fig. 1. The measurements were carried out for configurations of two elements with different heights, aligned in one line. The results of testing presented in this work relate to a fixed ratio of object height $\mathrm{H}_{1} / \mathrm{H}_{2}=0.6$ and three values of their "immersion" in boundary layer $\mathrm{H}_{2} / \delta=0.3,0.6$, and 1.0 .

Measurements of $\mathrm{CO}_{2}$ concentrations were taken in the gap between the elements in measurement cross-sections

a)

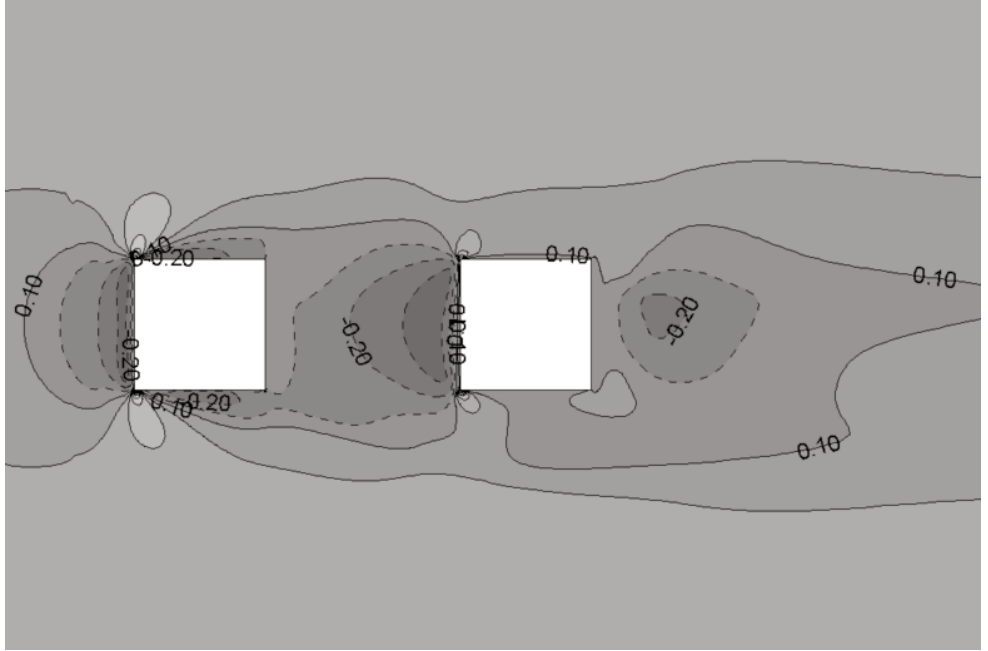

b)

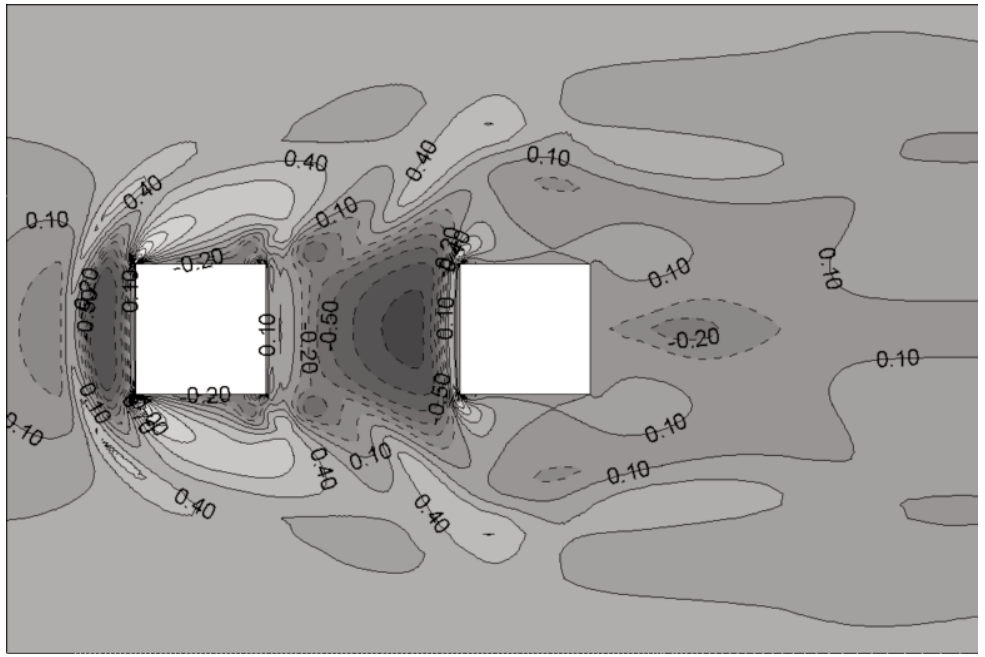

c)

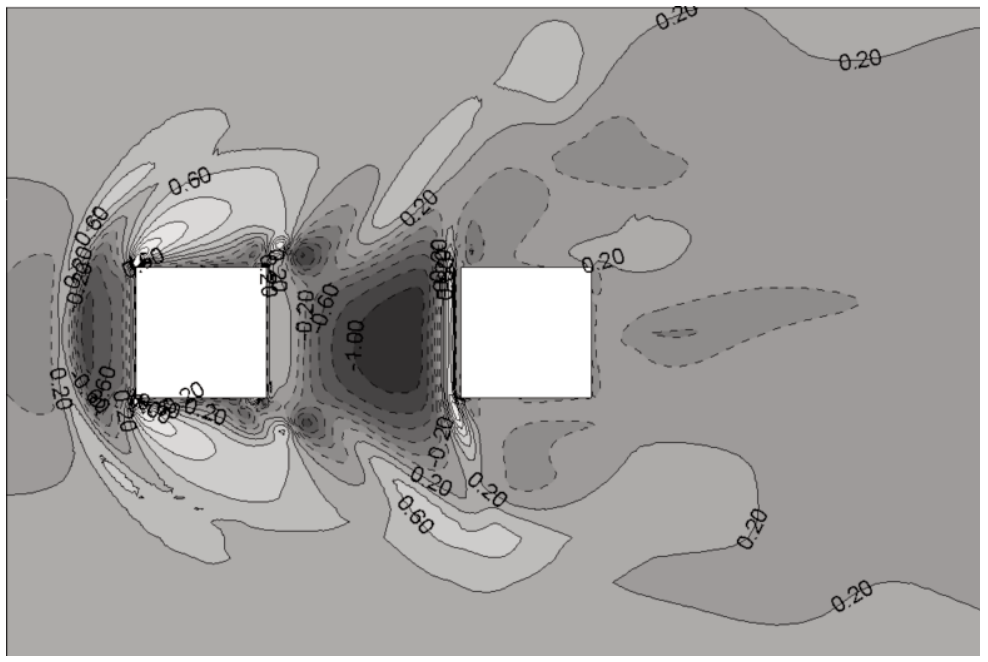

Fig. 3. The $x$-component of mean skin friction on the ground for the immersion parameter a) $\left.\mathrm{H}_{2} / \delta=0.3, b\right) \mathrm{H}_{2} / \delta=0.6$, and c) $\mathrm{H}_{2} / \delta=1$. 
$\mathrm{x}_{1} / \mathrm{D}=0.5,0.625,0.75,0.875,1$, and 1.25 - both in system axis and along the edges of external objects, for four different positions of emission source $\mathrm{z}_{\mathrm{S}}=0,0.5 \mathrm{H}_{1}, \mathrm{H}_{1}$, and $1.5 \mathrm{H}_{1}$ situated at $1.5 \mathrm{D}$ in front of the windward cube. In order to visualize the flow-modifying impact of the leeward object, some measurements of $\mathrm{CO}_{2}$ concentration profiles were also taken for a single one.

\section{Discussion of Results}

Analysis of the gas pollutant dispersion process requires in-depth identification of the structure of flow around the buildings. The flow structure around three-dimensional bluff-body located on the surface with formed boundary layer is characterized by a high level of complexity. In the presented spatial diagram of flow line (Fig. 2), the following characteristic areas in this type of flow can be distinguished: area of the horseshoe vortex forming in front of the object, upper flow, close and further wake zones. According to Hosker [14], flow around the object is composed of a range of separation and adherence points classified as singular nodal and saddle points. One can distinguish here a horseshoe vortex, whose name derives from its characteristic shape, and post-edge forties located a close distance from the rear side of the object.

Object impact zone, i.e. the area where the velocity field is strongly disturbed by the presence of the obstacle, changes considerably if another object is placed in the aerodynamic wake.

The case under consideration in this work concerns the arrangement characterized by $\mathrm{H}_{1} / \mathrm{H}_{2}$ parameter, which is conducive to the occurrence of the so-called "downwash" effect $\mathrm{H}_{1} / \mathrm{H}_{2}=0.6$. This effect consists of washing the front side of the leeward object with large air masses, which results in strong air circulation in the area between cuboids, which determines flow structure between them. This situation is presented in Fig. 3, which shows the result of numerical modeling of shear stress distribution on the ground surface as distribution of $\mathrm{x}$-component of mean skin friction $\left(\tau_{\mathrm{x}}[\mathrm{Pa}]\right)$. A rise in $\mathrm{H}_{2} / \delta$ parameter causes deeper effect of windward object to the front of the flow. Disturbing the impact of the second object, expressed particularly in location of the couple of post-edge vertices behind windward object and changes in the shape of lateral flow, is distinctly visible. The level of modification of flow around the analyzed arrangement of objects of tandem type depends on many factors. Change in height of the elements of the agreement impacts on changes in the immersion parameter in boundary layer. As results from Fig. 3, this parameter has significant impact on the flow structure.

The influence of flow pattern around two cuboids in canyon arrangement on the behavior of the skin friction distributions obtained as a result of numerical simulation is strongly marked in the space between cuboids and behind the second one. The biggest changes in flow field are observed in the area between objects. Rise in object height in relation to layer thickness causes rise in impact of wind- ward object and increase in width and length of recirculation zone and extension of the area taken by vortices. Aerodynamic influence of the leeward object is wider than the width of its horseshoe vortex of the windward object. Changes also concern the width of the zone, which for $\mathrm{H}_{2} / \delta=0.3$ considerably exceeds the line that is an extension of side surface of cuboids, whereas recirculation zone in $\mathrm{H}_{2} / \delta=1.0$ is considerably bigger than object width.

The observed modifying impact of interaction between the objects in tandem arrangement is reflected in the results of measurements of concentration of the tracer gas emitted in their environment.

From the perspective of pedestrians, the most interesting is the concentration of pollutants at ground level. The concentration of the tracer gas at ground level in the gap between objects for the emission source height $\mathrm{z}_{\mathrm{s}}=\mathrm{H}_{1}$ (at height of roof) is shown in Fig. 4.

As can be seen, the higher the windward object the lower the level of the tracer gas. A similar effect for a single object was observed by Lawton and Robins [15]. In the case of the arrangement being considered here, furthermore "downwash" effect was observed and, according to Fig. 3c, it is most intensive for $\mathrm{H}_{2} / \delta$ ratio equal to 1 . An additional relatively high object situated among the low-height elements can contribute to the intensification of mixing processes and consequently lead to improved air quality at the pedestrian level as pointed out by Vanweert and VanRooij [16]. Appropriate design of the wind environment with the presence of emission sources is very important, especially when taking into account human health and life comfort.

Configuration of the objects as well as location of the emission sources strongly influences pollutant dispersion. Maps of the gas tracer concentration for $\mathrm{H}_{2} / \delta=0.6$ and different positions of the emission source $\left(\mathrm{z}_{\mathrm{s}}=0,0.5 \mathrm{H}_{1}, \mathrm{H}_{1}\right.$, and $1.5 \mathrm{H}_{1}$ ) located at the same distance from the windward object $\left(x_{s}=-2 D\right)$ are shown in Fig. 5. In order to illustrate the modifying effect of the leeward object the maps of concentration for a single element, being placed identically as the windward object in tandem arrangement, were added.

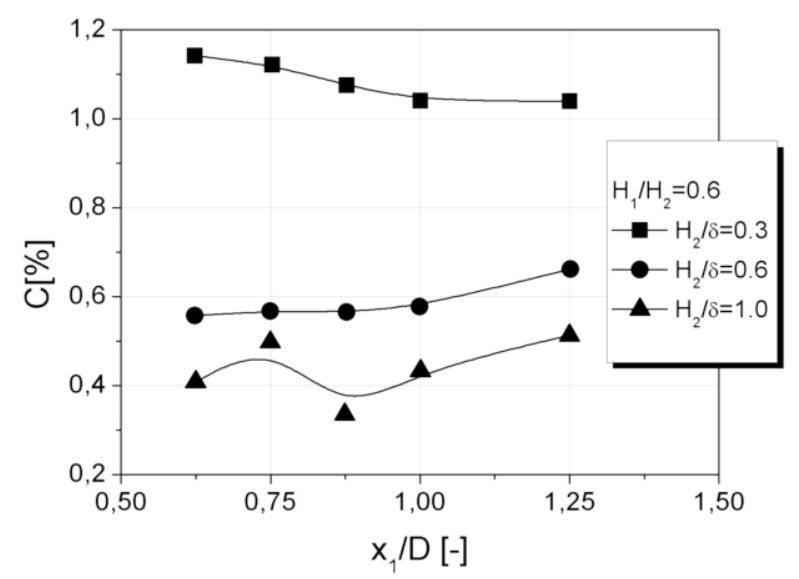

Fig. 4. Concentrations at ground-level on centreline for tandem arrangement with different immersion parameters $\mathrm{H}_{2} / \delta=0.3,0.6$, and 1 for emission source $z_{\mathrm{s}}=\mathrm{H}_{1}$. 

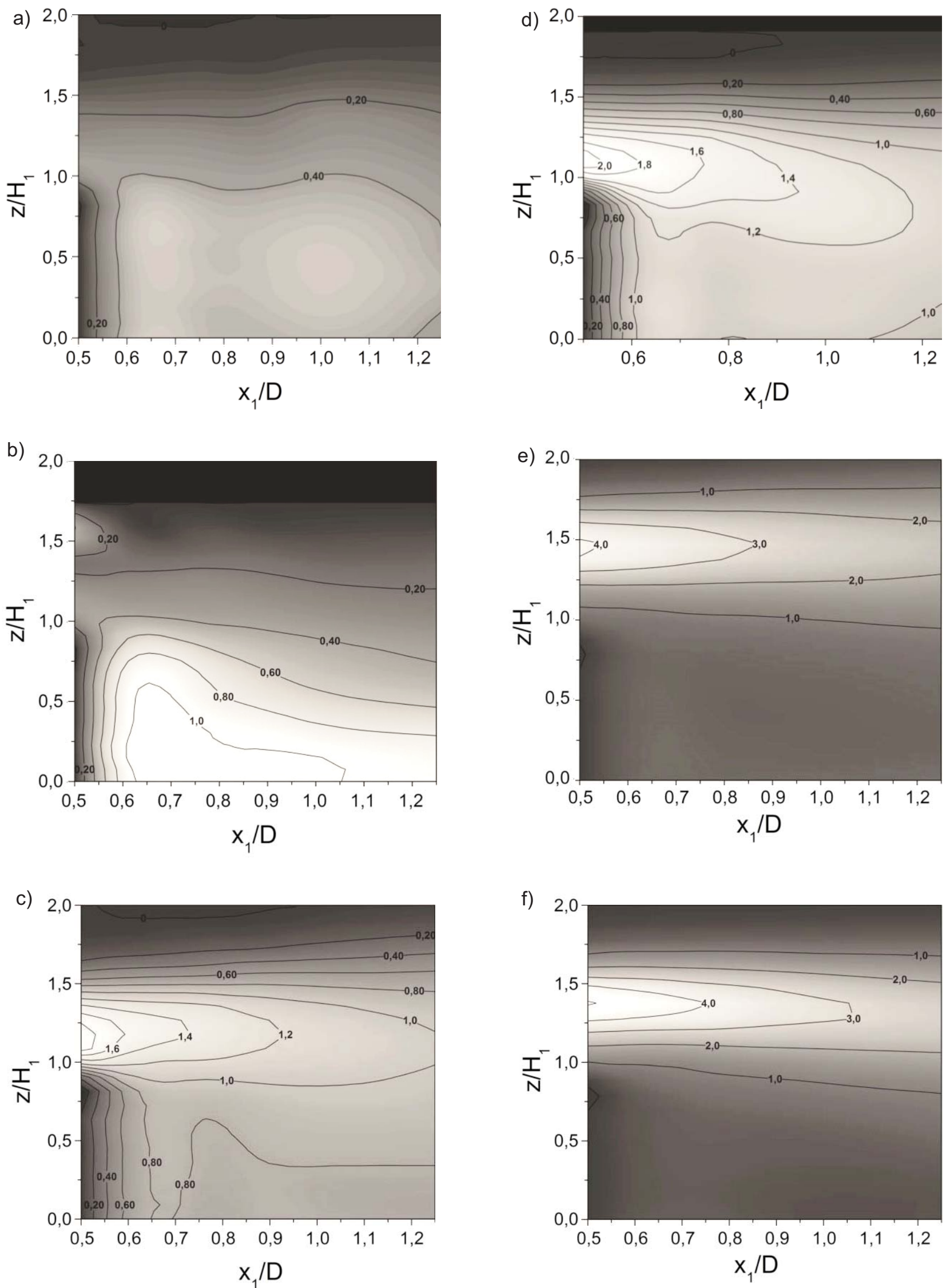

Fig. 5. Distribution of mean concentration $\mathrm{C}[\%]$ in the inter-obstacle gap for: a) tandem arranement $\mathrm{H}_{1} / \mathrm{H}_{2}=0.6$ and location of emission source $\mathrm{z}_{\mathrm{s}}=0$; b) single windward object and location of emission source $\mathrm{z}_{\mathrm{s}}=0$; c) tandem arranement $\mathrm{H}_{1} / \mathrm{H}_{2}=0.6$ and location of emission source $\mathrm{z}_{\mathrm{s}}=0.5 \mathrm{H}_{1} ;$ d) single windward object and location of emission source $\mathrm{z}_{\mathrm{s}}=0.5 \mathrm{H}_{1} ;$ e) tandem arranement $\mathrm{H}_{1} / \mathrm{H}_{2}=0.6$ and location of emission source $\mathrm{z}_{\mathrm{s}}=\mathrm{H}_{1} ; \mathrm{f}$ ) single windward object and location of emission source $\mathrm{z}_{\mathrm{s}}=\mathrm{H}_{1}$. 
g)
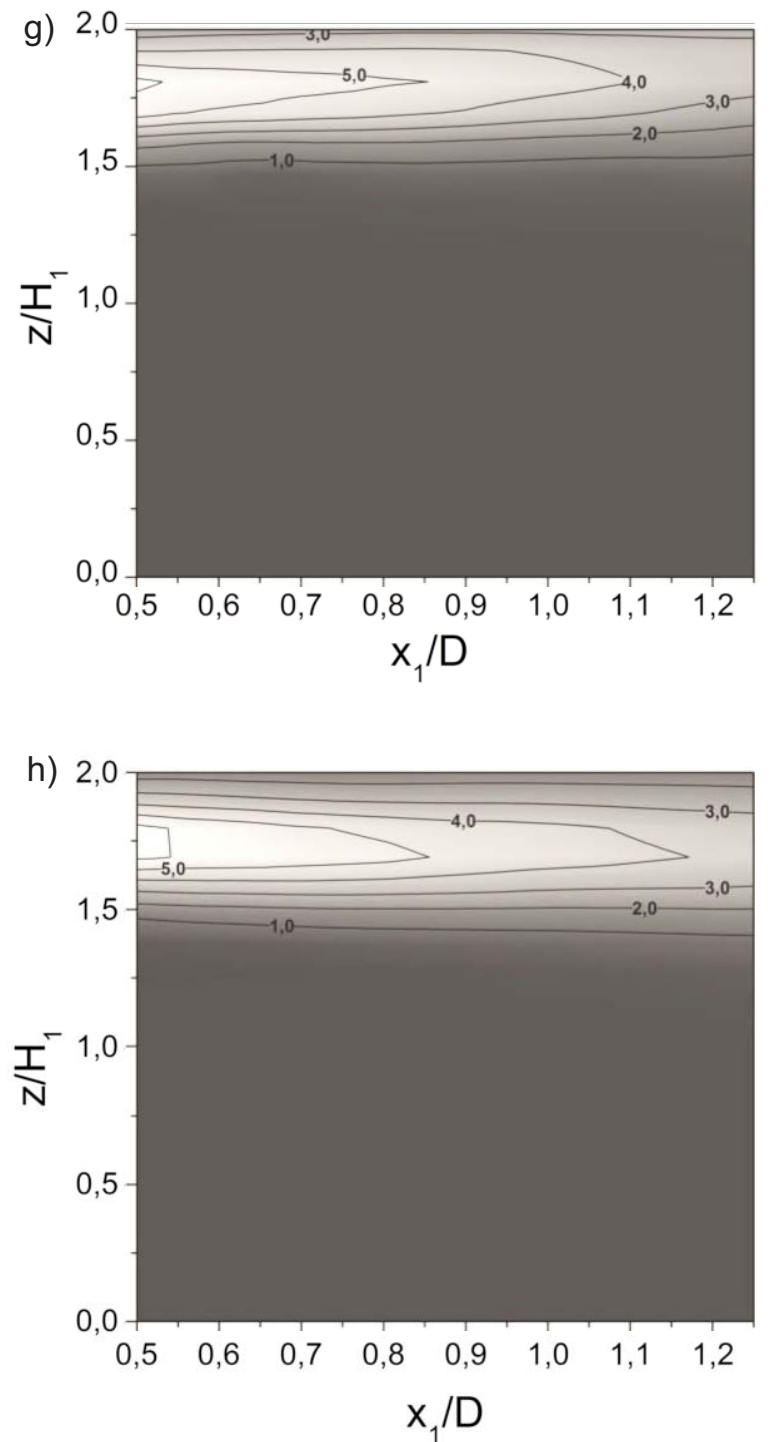

Fig. 5. Continued. Distribution of mean concentration $\mathrm{C}[\%]$ in the inter-obstacle gap for: g) tandem arranement $\mathrm{H}_{1} / \mathrm{H}_{2}=0.6$ and location of emission source $\mathrm{z}_{\mathrm{s}}=1.5 \mathrm{H}_{1} ; \mathrm{h}$ ) single windward object and location of emission source $\mathrm{z}_{\mathrm{s}}=1.5 \mathrm{H}_{1}$.

For different emission source heights, different distributions of the tracer gas concentration were obtained, which is an effect of various transport mechanisms. The highest qualitative and quantitative differences in the distributions of $\mathrm{CO}_{2}$ concentration are observed for $\mathrm{z}_{\mathrm{s}}=0$ (Figs. $5 \mathrm{a}$ and $5 b)$. In this case the tracer gas is mainly transported by the subsurface structure vortices and that is why maximum values of concentration are found at the ground level. It should be noted that the maximum values of $\mathrm{CO}_{2}$ concentration in the case of a single object are more than two times greater than in tandem arrangement configuration. According to Gnatowska [17], a disturbing effect of the second object causes the spanwise displacement of the location of the post-edge vortices pair behind the windward object and changes the lateral flow pattern. As can be seen, this leads to modification of the distribution of the tracer gas concentration. In the case of the arrangement when $z_{s}$ strives for height of the second building, we observed the relatively lower values of concentration C [\%] at the axis of symmetry $(y / D=0)$ in gap region when compared to the single object case as shown in Figs. $5 \mathrm{c}-\mathrm{h}$. Qualitative agreement is observed, although some quantitative discrepancies are present. For $\mathrm{z}_{\mathrm{s}}=0.5 \mathrm{H}_{1}$ (Figs. $5 \mathrm{c}-\mathrm{d}$ ) the maximum values of gas marker concentration in the array case equal $\mathrm{C}_{\max }=2 \%$, and for a single object $\mathrm{C}_{\max }=2.2 \%$, while for $\mathrm{z}_{\mathrm{s}}=\mathrm{H}_{1}$ (Figs. 5e-f), these values are $4.5 \%$ and $5.5 \%$, respectively. In addition, for $\mathrm{Z}_{\mathrm{s}}=0.5 \mathrm{H}_{1}$ the maximum concentration values are slightly above the height of the first object, while for $\mathrm{z}_{\mathrm{s}}=\mathrm{H}_{1}$ clearly above the height of the windward object. In both cases the modifying effect of the leeward object presence on the distribution of the tracer gas in the gap between objects is very clear, although it has different character. This may be explained by different transport mechanisms, i.e. for $\mathrm{z}_{\mathrm{s}}=0.5 \mathrm{H}_{1}$ gas marker is drifted by the upper and surface flow, while for $\mathrm{z}_{\mathrm{s}}=\mathrm{H}_{1}$ case $\mathrm{CO}_{2}$ transport is caused mainly by the upper flow. In the case of $z_{\mathrm{s}}=1.5 \mathrm{H}_{1}$ (Figs. $5 \mathrm{~g}$-h), the effect of leeward building presence is minimal, mainly because the $\mathrm{CO}_{2}$ stream is lifted over the object, thus the gas tracer concentration at ground level is $\mathrm{C}=0[\%]$.

The level of tracer gas at ground level (pedestrian region) in the axis of symmetry $(\mathrm{y} / \mathrm{D}=0)$ for different positions of emission sources for arrangement $\mathrm{H}_{1} / \mathrm{H}_{2}=0.6$ is shown in Fig. 6, except $\mathrm{z}_{\mathrm{s}}=1.5 \mathrm{H}_{1}$, for which $\mathrm{C}$ equals 0 $(\mathrm{C}=0[\%])$. For comparison, corresponding results for a single object $\left(\mathrm{H}=\mathrm{H}_{1}\right)$ are also presented. One may notice influence of the emission source height as well as the object configuration on the tracer gas distribution at ground level. The highest and similar values of $\mathrm{C}[\%]$ in the case of a single object were estimated for $\mathrm{z}_{\mathrm{s}}=0$ and $\mathrm{z}_{\mathrm{s}}=0.5 \mathrm{H}_{1}$, while the lowest values were found for $\mathrm{z}_{\mathrm{s}}=\mathrm{H}_{1}$. Only in the last case did the leeward object increase the level of $\mathrm{CO}_{2}$ concentration at the ground. In other cases, for tandem arrangement lower values than for a single object are observed, but more than twice reduction in concentration at the ground level is observed for $\mathrm{z}_{\mathrm{s}}=0$.

However, the problem of the location of emission sources and air intakes for ventilation systems in the case of existing or proposed buildings is not straightforward.

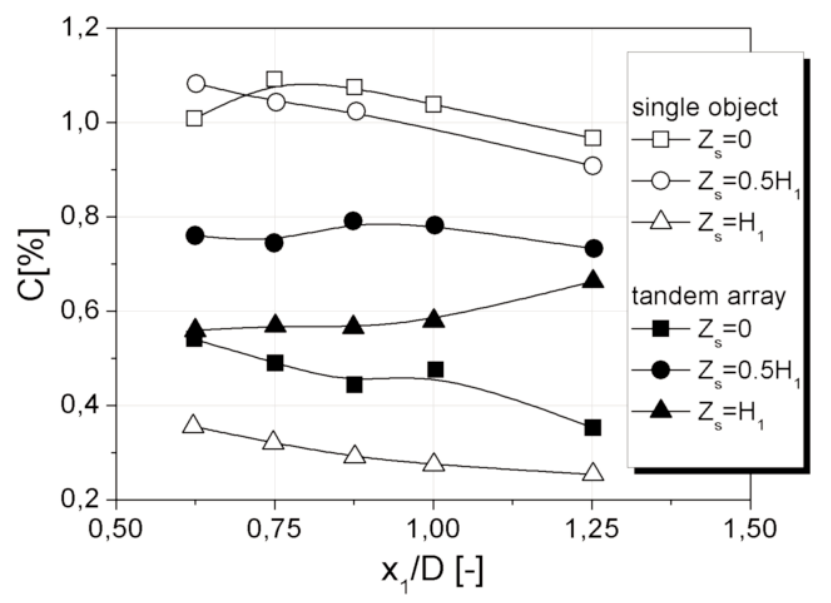

Fig. 6. Ground-level centreline concentrations for different heights of emission source $z_{\mathrm{s}}$. 


\section{Conclusions}

Wind comfort in built-up areas may be affected by a large range of parameters, mainly by wind velocity around building and adequate air quality. Those criteria are often in contradiction, because ensuring proper air quality requires adequate ventilation of built-up areas. Its effectiveness depends on wind direction, building configuration, and location of emission sources.

The performed experimental and numerical research was aimed primarily at the development of the existing knowledge of the interaction between objects located on the ground and its influence on pollutant dispersion. Such studies may contribute to the better understanding of physical processes and provide necessary information for the development of numerical modeling.

The presented results showed that the appropriate location of buildings and the emission sources is very important, especially when taking into account human health and wind comfort. The choice of emission source shape and size may positively influence pollutant dispersion emitted in building environments and in turn may lead to improved air quality at the pedestrian level.

\section{Acknowledgements}

The investigations presented in this paper have been obtained within project No. BS/MN-1-103-301/11/P: "Modeling of flow phenomena and wind comfort in builtup areas."

\section{References}

1. BLOCKEN B., STATHOPOULOS T., CARMELIET J. Wind environmental conditions in passages between two long narrow perpendicular buildings. J. Aerospace Eng. 21, (4), 280, 2008.

2. CHANG CH., LIN J.S., CHENG C.M., HONG YS. Numerical simulations and wind tunnel studies of pollutant dispersion in the urban street canyons with different height arrangements. Journal of Marine Science and Technology 21, (2), 119, 2013.

3. MAJEWSKI G., PRZEWOŹNICZUK W. Study of particulate matter pollution in Warsaw area. Pol. J. Environ. Stud., 18, (2), 293, 2009.
4. BOKWA A. Environmental impacts of long-term air pollution changes in Krakow. Pol. J. Environ. Stud., 17, (5), 673, 2008.

5. MACIAS A., GADZIŃSKI J. Assessment of road transport environmental impact as illustrated by a metropolitan area Pol. J. Environ. Stud., 22, 6, 1749, 2013.

6. MORYN-KUCHARCZYK E., GNATOWSKA R. Pollutant dispersion in flow around bluff bodies arrangement. Wind Energy, Springer Verlag Berlin Heidelberg pp. 49-53, 2007.

7. LATEB M., MASSON C., STATHOPOUlOS T., BÉDARD C. Numerical simulation of pollutant dispersion around a building complex. Build. Environ. 45, 1788, 2010.

8. BLOCKEN B., STATHOPOULOS T., CARMELIET J., HENSEN J. Application of CFD in building performance simulation for the outdoor environment: an overview. J Build Perform Simulation 4, (2), 157, 2011.

9. TOMINAGA Y., STATHOPOULOS T. Numerical simulation of dispersion around an isolated cubic building: model evaluation of RANS and LES. Build. Environ. 45, 2231, 2010.

10. GOUSSEAU P., BLOCKEN B., VAN HEIJST G.J.F. CFD simulation of pollutant dispersion around isolated buildings: on the role of convective and turbulent mass fluxes in the prediction accuracy. J. Hazard. Mater. 194, 422, 2011.

11. TOMINAGA Y., STATHOPOULOS T. CFD modeling of pollution dispersion in building array: evaluation of turbulent scalar flux modeling in RANS model using LES results. J. Wind Eng. Ind. Aerod. 104-106, 484, 2012.

12. LATEB M., MASSON C., STATHOPOULOS T., BEDARD C. Effect of Stack Height and Exhaust Velocity on Pollutant Dispersion in the Wake of a Building. Atmos. Environ. 45, (29), 5150, 2011.

13. WONG P.T.Y., KO N.W.M., CHIU A.Y.W. Flow characteristics around two parallel adjust square cylinder of different sizes. J. Wind Eng. Ind. Aerod. 54-55, 263, 1995.

14. HOSKER P.R. Jr. Empirical estimation of wake cavity size behind block-type structures, Proc. $4^{\text {th }}$ Symposium on Turbulence, Diffusion and Air Pollution, Reno Nevada, American Meteorological Society, Boston Mass, pp. 603609, 1979.

15. LAWTON T., ROBINS A.G. Flow and dispersion around buildings. Proc. PHYSMOD 2007, Orleans/France, pp. 203206, 2007.

16. VAN WEERT F.L.H., VAN ROOIJ J.I.J.H. Air-quality and spatial planning. Proc. PHYSMOD 2007, Orleans/France, pp. 191-196, 2007.

17. GNATOWSKA R. Aerodynamic Characteristics of ThreeDimensional Surface-Mounted Objects in Tandem Arrangement. Int. J. Turbo Jet Eng. 28, 1, 21, 2011. 
